\title{
Postnatal Development of Subcallosal Zone Following Suppression of Programmed Cell Death in Bax-deficient Mice
}

\author{
Woon Ryoung Kim and ${ }^{\dagger}$ Woong Sun \\ Department of Anatomy, Korea University College of Medicine, Seoul 136-705, Republic of Korea
}

\begin{abstract}
Neural stem cells are found in adult mammalian brain regions including the subgranular zone (SGZ) of the dentate gyrus (DG) and the subventricular zone (SVZ). In addition to these two regions, other neurogenic regions are often reported in many species. Recently, the subcallosal zone (SCZ) has been identified as a novel neurogenic region where new neuroblasts are spontaneously generated and then, by Bax-dependent apoptosis, eliminated. However, the development of SCZ in the postnatal brain is not yet fully explored. The present study investigated the precise location and amount of neuroblasts in the developing brain. To estimate the importance of programmed cell death (PCD) for SCZ histogenesis, SCZ development in the Bax-knockout (KO) mouse was examined. Interestingly, an accumulation of extra neurons with synaptic fibers in the SCZ of Bax-KO mice was observed. Indeed, Bax-KO mice exhibited enhanced startle response to loud acoustic stimuli and reduced anxiety level. Considering the prevention of PCD in the SCZ leads to sensory-motor gating dysfunction in the Bax-KO mice, active elimination of SCZ neuroblasts may promote optimal brain function.
\end{abstract}

Key words : Subcallosal zone, Adult neurogenesis, Bax, Cell death, Neuroblast

\section{INTRODUCTION}

Over the last half of century, genetic and cellular properties of neural stem cells (NSCs) in the adult brain have been identified, and many reports have consistently addressed the adult neurogenesis in the subgranular zone (SGZ) of the dentate gyrus (DG) and the subventricular zone (SVZ) in many mammalian species, including humans (Eriksson et al., 1998; Sanai et al., 2004; Kim et al., 2011; Kim et al., 2012a). Considering the limited use of stem cells for therapeutic transplantation, the presence of NSCs in the adult brain has raised hopes for the promotion of spontaneous brain regeneration. Supporting this, injury-induced neurogenesis and neuronal migration toward injured areas have been reported. For example, focal ischemia, or traumatic brain injury, promotes cell proliferation in the neurogenic regions (Schmidt \& Reymann, 2002; Bendel et al., 2005; Koketsu et al., 2006; Yu et al., 2008; Kernie \& Parent, 2010). However, the activation of endogenous neurogenesis depends on the extent and the location of the brain injury (Kim et al., 2011), and the identification of novel neurogenic regions containing NSCs should provide additional potential for neuronal repair in nonneurogenic regions such as the cerebral cortex.

Recently, the present authors and others have identified the subcallosal zone (SCZ) as a novel adult neurogenic region (Seri et al., 2006; Kim et al., 2011). The SCZ is located between the cerebral cortex and the hippocampus in the posterior brain regions. Interestingly, when the programmed cell death (PCD) of neurons was blocked

\footnotetext{
Manuscript received 16 July 2013, Received in revised form 1 August 2013, Accepted 5 August 2013

${ }^{\dagger}$ Corresponding Author : Woong Sun, Department of Anatomy, Brain Korea 21, Korea University College of Medicine, Anam-dong, Sungbuk-gu, Seoul 136-705, Republic of Korea. Tel. : +82-2-920-6404, Fax : +82-2-929-5696, E-mail : woongsun@korea.ac.kr

This is an Open Access article distributed under the terms of the Creative Commons Attribution Non-Commercial License(http://creativecommons.org/ licenses/by-nc/3.0) which permits unrestricted non-commercial use, distribution, and reproduction in any medium, provided the original work is properly cited.
} 
by the elimination of the pro-apoptotic gene, Bax, many neuroblasts were accumulated in the SCZ, indicating that there is spontaneous production along with massive loss of young neurons in the SCZ (Kim et al., 2011). Considering that the SCZ is anatomically close to the corpus callosum and cerebral cortex, it was hypothesized in the present study that the spontaneous neurogenesis and PCD in the SCZ may have functional significance for the corpus callosum related to the cerebral cortex. In addition, although brain injury stimulates cell proliferation and migration toward the injured site, the early postnatal development and distribution of the SCZ neuroblasts remains unclear. Thus, the present study further investigated the distribution of neuroblasts in early postnatal and adult SCZ in wild type and Bax-KO mice. Moreover, for the comprehensive understanding of the function of SCZ neurogenesis, the cortex-related behavioral functions of Bax-KO mice were tested to address whether prevention of the spontaneous PCD in the SCZ interfered with cerebral cortex function.

\section{MATERIALS AND METHODS}

\section{Animals}

Bax-KO mice were maintained as heterozygotes on a C57Bl/6 background. Sibling animals were collected and individually genotyped by PCR as previously described (Kim et al., 2011). For BrdU labeling, postnatal day 3 (P3) mouse pups received single injections of BrdU (50

/g body weight) intraperitoneally, and the animals were sacrificed 4-weeks following the BrdU injection. All experiments were carried out in accordance with the ethical guidelines of Korea University and with the approval of the Animal Care and Use Committee of Korea University.

\section{Histology}

Immunohistochemical analyses were performed as previously reported (Song \& Cheon, 2011). Briefly, mice were deeply anesthetized and perfused with $0.9 \%$ saline and $4 \%$ paraformaldehyde solution. Following overnight post-fixation in the same fixative, brains were cryoprotected in $30 \%$ sucrose solution. Brains were sectioned and collected serially (40 thickness) and stored on 50\% glycerol $/ 50 \%$ PBS solution at $-20^{\circ} \mathrm{C}$ until use. For BrdU staining, sections were pre-treated with $0.2 \mathrm{~N} \mathrm{HCl}$ for 1 h at $37^{\circ} \mathrm{C}$, washed with PBS, and blocked with 3\% BSA and $0.1 \%$ Triton X-100 in PBS for $30 \mathrm{~min}$. Primary antibodies were applied overnight: anti-DCX (1:1,000; Santa Cruz Biotechnology), anti-BrdU (AbDSerotec), antiSV2 (HDB), anti-SV2 (HDB). After several washes with PBS, appropriate secondary antibodies were applied for 30 min. Subsequently, sections were washed, mounted, and observed using a confocal microscope (Zeiss LSM510 and 700, Goettingen, Germany).

\section{Acoustic startle response test}

Mice received the test in a transparent plastic restrainer (5 $\mathrm{cm}$ diameter $\times 10 \mathrm{~cm}$ ) situated in a sound-attenuating chamber with background noise of $65 \mathrm{~dB}$. Startle responses were measured by load cell, amplified, and stored into the computer (LabVIEW). Before the start of the experiment, mice were acclimated in the restraints for 20 minutes. The mice receive no stimuli except a $65 \mathrm{~dB}$ background noise during a 5 min acclimation period. At the beginning of the experiment, mice were exposed to $100 \mathrm{~dB}$ startle stimuli (SS) three times, since the early responses to the SSs are quite different from the responses throughout the whole experiment. Subsequently, 88 trials were conducted, which consisted of pseudo-random delivery of 11 kinds of the acoustic stimuli: $70 \mathrm{~dB}$ to $120 \mathrm{~dB}$ with $5 \mathrm{~dB}$ intervals. All trials were presented with an average ITI of $15 \mathrm{~s}$ (range 5-30 s). Data were collected for 65 ms from the onset the startle stimulus and the maximum response during the 65 ms-period was used in the analysis.

\section{Open field test}

The test arena was a gray Plexiglas arena $(50 \times 50 \mathrm{~cm})$ enclosed by walls (25 cm height) and illuminated by a dim light. Mice were placed in a center, and their movements were recorded for 10 min by a tracking system 
(SmarTrack). The time spent in the center square $(30 \times 30$ $\mathrm{cm}$ ) was recorded. The ratio of the time spent in the center to $10 \mathrm{~min}$ was calculated and used as a measure of anxiety-related behavior.

\section{Elevated plus maze}

The arena of the elevated plus maze (EPM) had the shape of a plus sign with four 30-cm-long and 5-cm-wide arms, connected by a $5 \times 5 \mathrm{~cm}$ center area. Two opposing arms were bordered by $25 \mathrm{~cm}$ high walls (closed arms), whereas the other two arms (open arms) were bordered by a $0.3 \mathrm{~cm}$ rim. The plus was elevated $75 \mathrm{~cm}$ from the floor. The mouse was placed in the center facing an open arm and observed for $10 \mathrm{~min}$. The observer measured the time spent in the open and closed arms (calculated as when all the four paws were on an arm). This parameter was taken as measuresof anxiety-related behavior.

\section{Statistical analysis}

Statistical significance of the experiments was evaluated using two-tailed $(\alpha=0.05)$ one-way repeated-measures ANOVA with Scheffe's multiple comparison (startle response test) and $t$-test (open field test, EPM). All analyses were performed using SPSS software (Chicago, IL), and all values are given as the mean \pm standard error of the mean (SEM).

\section{RESULT}

\section{Postnatal development of the mouse SCZ}

In the 15 day-old wild-type (WT) mouse brain, many neuroblasts expressing doublecortin (DCX) were found along with the lateral and dorsal aspects of the SVZ of lateral ventricle (Fig. 1A, c, red), Therefore, the SVZ and the SCZ were closely positioned at this stage, and many densely clustered DCX-expressing neuroblasts were found in the SCZ (Fig. 1A, e), suggesting that SCZ NSCs are originated from the posterior extension of the SVZ lateral wall. In this stage, a few neuroblasts were also found in the primary dentate matrix (Fig. 1A, d), which

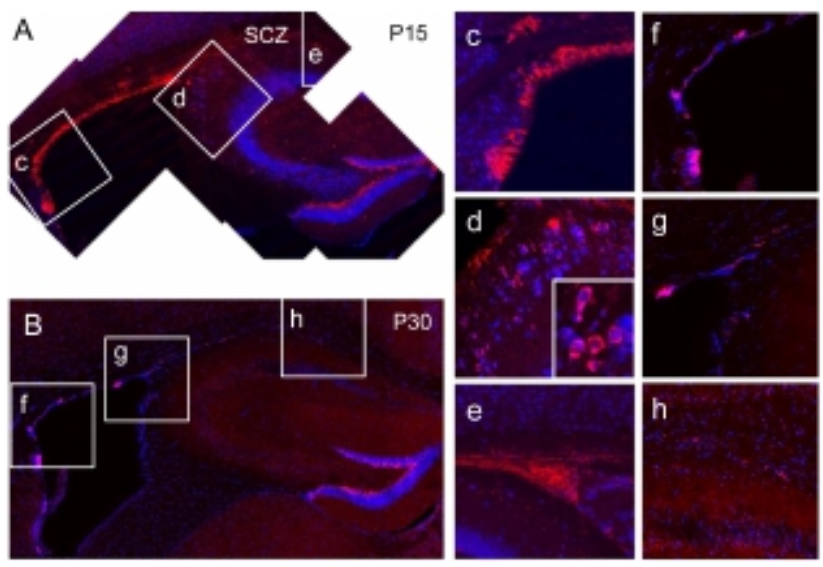

Fig. 1. Distribution of DCX expressing neuroblasts (red) at postnatal day $15(\mathrm{~A}, \mathrm{c}-\mathrm{e})$ and day $30(\mathrm{~B}, \mathrm{f} \mathbf{H})$. Enlarged image of inset in A and B, lateral ventricular wall (c, f), primary dentate matrix $(\mathrm{d}, \mathrm{g})$, subcallosal zone $(\mathrm{e}, \mathrm{h})$. LV: lateral ventricle, GCL: granule cell layer, SCZ: subcallosal zone.

is an origin of DG progenitors and granule cells (Fig. 1A). However, with further separation of the SVZ and SCZ, the amount of neuroblasts appeared to be reduced both in the SVZ (Fig. 1B, f) and SCZ (Fig. 1B, h) at P30 (Fig. 1B, $\mathrm{f}-\mathrm{h}$ ). In addition, DCX-expressing neuroblasts completely disappeared in the primary dentate matrix (Fig. 1B, g). Therefore, it appears that the neuroblasts are actively produced during the early postnatal development. However, with reduction of neurogenesis in surrounding regions, proliferation of SCZ stem cells appears to be reduced progressively.

2. Programmed cell death of early postnatally generated neuroblasts in the $\mathrm{SCZ}$

Considering that there is virtually no mature neuron in the WT SCZ, the neuroblasts produced during the postnatal stage should be eliminated. Consistently with this, the present authors previously reported the massive PCD of neuroblasts in the SCZ, which are dependent on the Bax function (Kim et al., 2011). To trace the fate of early postnatally produced neurons, newly produced cells were selectively labeled by single injection of BrdU at P3, and the survival of these BrdU-labeled cells on P30 was examined (Fig. 2). In WT, whereas many 


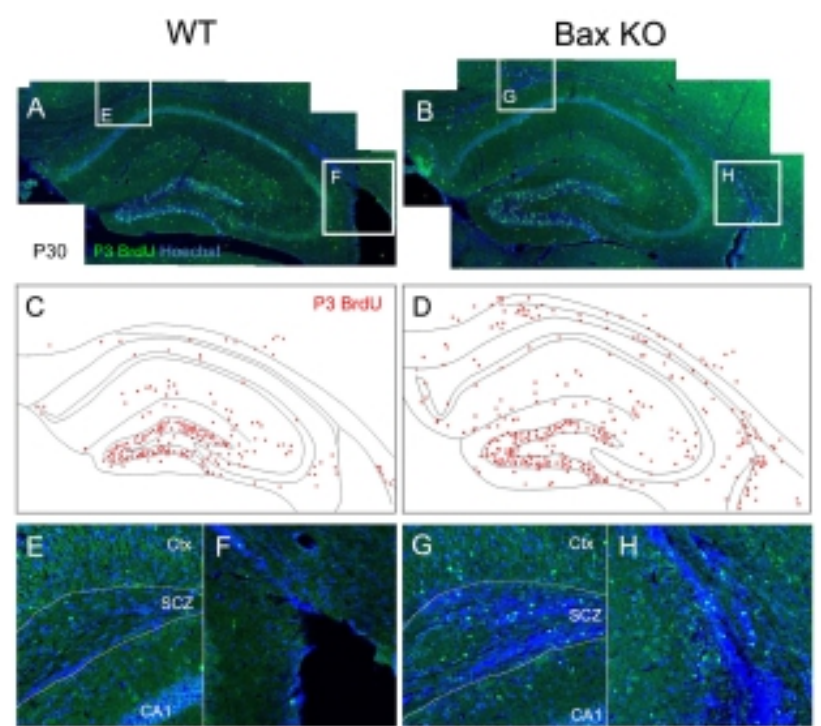

Fig. 2. Distribution of mature neurons in the adult (2 monthsold) SCZ. (A) Distribution of BrdU labeled neurons which born at postnatal day 3 (green) in the adult WT mouse and (B) Bax-KO mouse. $(\mathrm{C}-\mathrm{D}) \mathrm{BrdU}^{+}$cells are depicted by red dots. (E) BrdU ${ }^{+}$cells in the WT medial SCZ, (F) lateral SCZ, (G) in the Bax-KO medial SCZ, (H) lateral SCZ.

BrdU-labeled cells remained in the DG and molecular layers, BrdU-labeled cells were seldom found in the SCZ (Fig. 2, A, C, E, F). On the other hand, the Bax-KO SCZ contained many BrdU labeled cells (Fig. 2, B, D, $\mathrm{G}, \mathrm{H})$, suggesting that a large amount of newly produced cells during the early postnatal stages were eliminated by Bax-dependent PCD within the SCZ.

\section{Synaptic integration of Bax KO-rescued SCZ} neurons

Because a subset of SCZ cells in the Bax-KO can differentiate into mature neurons (Kim et al., 2011), here we examined the maturation of synapse formation within the SCZ of Bax-KO mice at two and six months. Using immunostaining method, we compared the expression of synaptic vesicle protein 2 (SV2), which is important for the neural transmission, in the Bax-KO SCZ (Fig. 3AD). However, significant SV2 immunoreactivity in the SCZ of WT mice (Fig. 3A, B), was not identified; many cells in the 6-month-old Bax-KO SCZ were immunoreactive

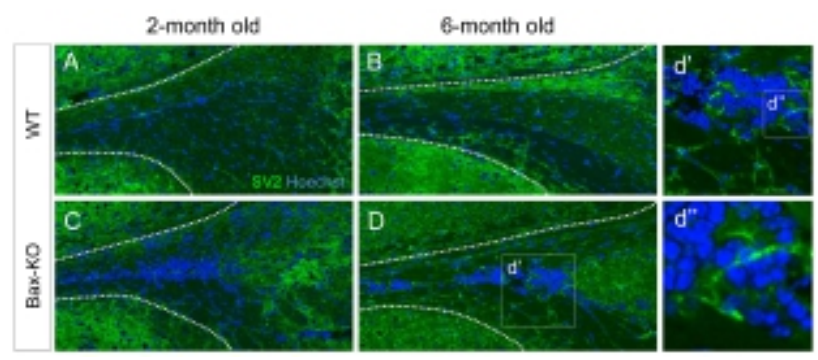

Fig. 3. SV2 expressing synaptic SCZ neurons in young (2 monthsold) mouse (A, C) and aged (6 month-old) mouse (B, D). There is no SV2 expressing cell in the WT SCZ (A, B); however SV2 expressing cells are increased in aged BaxKO mice (D) compared to young Bax-KO mice (C). Enlarged image of inset in D is d' and of inset in d' is d'.

for SV2 (Fig. 3C, D, d' and d'). On the other hand, 2-month-old Bax-KO SCZ had fewer SV2 immunoreactivities. Therefore, although initial neurogenesis occur in early postnatal stages, their maturation and the synaptic integration may require longer periods ( $>2$ months) in Bax-KO mice.

\section{Cerebral cortex-related behaviors of Bax-KO mice}

Because the SCZ is located between corpus callosum, excess neurons within the Bax-KO SCZ may affect brain function related with corpus callosum or corticostriatal connection. Thus, the functional alteration in the aged Bax-KO mice was examined. It is well known that the abnormal corpus callosum and related cerebral cortex lead to a deficit of sensory-motor gating (Ballmaier et al., 2007; John et al., 2008; Marcano-Reik \& Blumberg, 2008). Thus, we used acoustic stimulated startle reflex test to evaluate the sensory-motor gating function in Bax-KO mice. When various acoustic stimuli were applied to 2-month-old and 6-month-old mice, it was found that the startle response of the latter but not the former was significantly higher from $100 \mathrm{~dB}$ stimuli compared to the WT littermates (Fig. 4A, B). In addition, anxiety level is known to be affected by abnormal connection in corpus callosum (Yang et al., 2009). Thus, anxiety level of Bax-KO mice was also examined. Similarly to the startle responses, only aged (6-month-old) Bax-KO mice spent more time in the center of square open arena than WT littermates (Fig. 4C). Furthermore, in the EPM tests, 


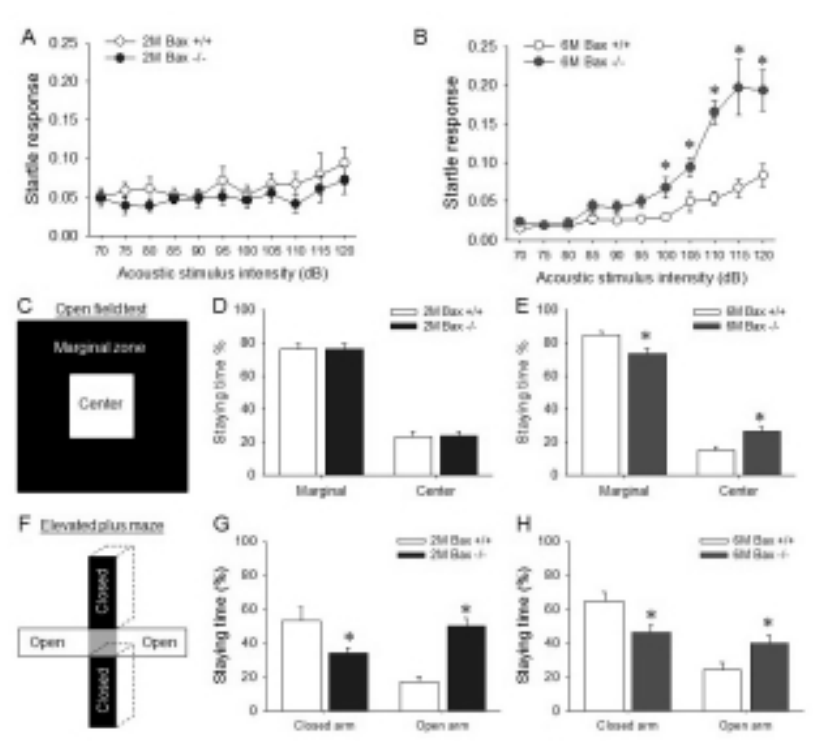

Fig. 4. Behaviors of Bax-KO mice. (A, B) Acoustic startle reflex test in young mouse (A) and aged mouse (B). Mean \pm SEM, ${ }^{*} p<0.05$, One-way repeated measured ANOVA. $(\mathrm{C}-\mathrm{E})$ open field test in young group $(\mathrm{B})$ and aged group (C). $(\mathrm{F}-\mathrm{H})$ Elevated plus maze test in young group (E) and aged group (F). Mean \pm SEM, ${ }^{*} p<0.05$, $t$-test.

both young and aged Bax-KO spent longer time in open arms compared to the WT littermates (Fig. 4E, F). These two results suggest that the anxiety level of Bax-KO mice was significantly decreased. Collectively, these results suggest that Bax-KO mice exhibit SCZ-dependent behavioral defects.

\section{DISCUSSION}

The SCZ is located between the hippocampus and the corpus callosum, which is compacted with fiber bundles. Embryonically, along with the hippocampal development, the caudal extremity of the posterior horn of the lateral ventricle is progressively narrowed from E14.5 (Rickmann et al., 1987). At the end, the wall of posterior lateral ventricle is bent above the hippocampus and enfolded with corpus callosum around E18.5 in rodent. Finally, the narrow posterior lateral ventricle is completely collapsed after birth. Considering the collapsed posterior LV become SCZ, the SCZ and SVZ are anatomically continuous, and the progenitors in these regions may share many similarities (Doetsch \& Alvarez-Buylla, 1996). Supporting this, both SCZ and SVZ-derived NSCs showed similar potency to produce neurons in vitro (Seri et al., 2007). Furthermore, the preliminary analysis of transcriptome by microarray indicated that $>99 \%$ of their gene expression profiles were similar (data not shown). As SVZ NSCs produces olfactory bulb neurons during the early postnatal development, it is reasonable to speculate that nearly SCZ NSCs also have similar proliferative potentials. Supporting this, it was found that early postnatal SCZ exhibited strong neurogenic properties. Even though these two NSC populations were globally similar and both regions produce massive neuroblasts, most neuroblasts generated from SCZ die, whereas neuroblasts from SVZ normally migrate to the olfactory bulb, and a large proportion (>50\%) of them survive (Kirschenbaum et al., 1999; Kim et al., 2007). Considering that SCZ is anatomically encapsulated by white matter, neurons in this area do not normally have an exit for cell migration or axonal projections. It is known that the survival of immature neurons is highly dependent on their ability to form synaptic connections with target neurons and ability to obtain sufficient tropic signals. The present authors have previously demonstrated that the Bax gene is essential for the execution of PCD during the provisional synaptic formation stages (Jin et al., 2003; Sun et al., 2004; Kim et al., 2007; Kim et al., 2009; Kim et al., 2011). Considering that PCD in the SCZ is also Bax-dependent, these results suggest that the inability of neuroblasts in the SCZ can cause massive PCD.

It is as yet unclear as to the biological function of spontaneous production of neuroblasts in the SCZ, because most produced neuroblasts are eliminated via Bax-dependent apoptotic pathways. Thus, to assess the significance of PCD of the SCZ neuroblasts, the present study examined the functional alteration of Bax-KO mice, which have the survived SCZ neurons. It was hypothesized that survived SCZ neurons in Bax-KO mice might form synaptic connections with extra-SCZ neurons and affect normal brain functions. Interestingly, it was found that 
the sensory-motor gating function was severely impaired in the aged Bax-KO mice. It has been reported that abnormal corpus callosum leads to a deficit of sensorymotor gating (Ballmaier et al., 2007; John et al., 2008; Marcano-Reik \& Blumberg, 2008), suggesting that the presence of SCZ neurons could negatively influence the corpus callosum-dependent brain functions. Interestingly, these behavioral alterations were significant only in the 6-month-old Bax-KO mice, even though accumulation of neuroblasts in the SCZ in Bax-KO mice was also prominent in 2-month-old mice. It was found that synaptic innervations of these survived neurons in Bax-KO mice appeared to take long periods, and the age-dependent increase in the SV2-immunoreactivities in the Bax-KO SCZ was observed. Accordingly, it was reasoned that SCZ-dependent phenotypes could be age-dependent. The impairment of corpus callosum is also associated with alterations in anxiety levels (Yang et al., 2009). When the postnatal lesion was given to corpus callosum, anxiety level was also reported to be decreased in the EPM test (Yang et al., 2009). Accordingly, it was found that the anxiety levels of Bax-KO mice were significantly lower than those of WT littermates, as revealed by two different behavioral test: open field and EPM tests. These phenotypes were found in the young (2-month-old) Bax-KO mice, raising the possibility that anxiety-related behaviors are more sensitive to the impairment of SCZ. Collectively, these results indicate that accumulation of ectopic neurons in the SCZ may impair the corpus callosum-related behaviors. Therefore, active elimination of the neuroblasts in the SCZ appears to be necessary for the maintenance of normal brain functions. However, the possibility that the behavioral alterations in Bax-KO mice are associated with the impairment of other brain regions, such as hippocampus (Kim et al., 2009) and olfactory bulb (Kim et al., 2007; Kim \& Sun, 2013), cannot be completely excluded. Further comprehensive analyses of the behaviors and the anatomical correlations are required to clarify this issue.

Currently, the significance of neuroblast formation in the SCZ is unclear. However, SCZ NSCs can produce neurons following brain injury. Various methods for the amplification of those aNSC population, directional differentiation, and promotion of survival within the pre-existed mature neurons have been investigated following brain injury (Schmidt \& Reymann, 2002; Bendel et al., 2005; Koketsu et al., 2006; Yu et al., 2008; Kernie \& Parent, 2010). Once the cortex is injured, not only SVZ and DG (Dash et al., 2001; Kernie et al., 2001; Kernie \& Parent, 2010), but also the SCZ neuroblasts are significantly increased (Kim et al., 2011). Thus, the SCZ stem cells may be a useful source for supplying neurons and/or glia when it is necessary.

\section{ACKNOWLEDGEMENTS}

This research was supported by Basic Science Research Program through the National Research Foundation of Korea (NRF) funded by the Ministry of Education, Science and Technology: NRF-2010-0020237 (WS), NRF-20110019212 (WS), NRF-2012M3A9C6049933 (WS), NRF2012M3A9C6049933 (WRK).

\section{REFERENCES}

Ballmaier M, Bortolato M, Rizzetti C, Zoli M, Gessa G, Heinz A, Spano P (2007) Cannabinoid receptor antagonists counteract sensorimotor gating deficits in the phencyclidine model of psychosis. Neuropsychopharmacology 32:2098-2107.

Bendel O, Bueters T, von Euler M, Ove Ogren S, Sandin J, von Euler G (2005) Reappearance of hippocampal CA1 neurons after ischemia is associated with recovery of learning and memory. J Cereb Blood Flow Metab 25:1586-1595.

Dash PK, Mach SA, Moore AN (2001) Enhanced neurogenesis in the rodent hippocampus following traumatic brain injury. J Neurosci Res 63:313-319.

Doetsch F, Alvarez-Buylla A (1996) Network of tangential pathways for neuronal migration in adult mammalian 
brain. Proc Natl Acad Sci USA 93:14895-14900.

Eriksson PS, Perfilieva E, Bjork-Eriksson T, Alborn AM, Nordborg C, Peterson DA, Gage FH (1998) Neurogenesis in the adult human hippocampus. Nat Med 4:13131317.

Jin K, Sun Y, Xie L, Peel A, Mao XO, Batteur S, Greenberg DA (2003) Directed migration of neuronal precursors into the ischemic cerebral cortex and striatum. Mol Cell Neurosci 24:171-189.

John JP, Shakeel MK, Jain S (2008) Corpus callosal area differences and gender dimorphism in neurolepticnaive, recent-onset schizophrenia and healthy control subjects. Schizophr Res 103:11-21.

Kernie SG, Erwin TM, Parada LF (2001) Brain remodeling due to neuronal and astrocytic proliferation after controlled cortical injury in mice. J Neurosci Res 66:317-326.

Kernie SG, Parent JM, (2010) Forebrain neurogenesis after focal ischemic and traumatic brain injury. Neurobiol Dis 37:267-274.

Kim WR, Christian K, Ming GL, Song H (2012a) Timedependent involvement of adult-born dentate granule cells in behavior. Behav Brain Res 227:470-479.

Kim WR, Chun SK, Kim TW, Kim H, Ono K, Takebayashi H, Ikenaka K, Oppenheim RW, Sun W (2011) Evidence for the spontaneous production but massive programmed cell death of new neurons in the subcallosal zone of the postnatal mouse brain. Eur J Neurosci 33:599-611. Kim WR, Kim JY, Moon Y, Kim HJ, Kim H, Sun W (2012b) Regional difference of reactive astrogliosis following traumatic brain injury revealed by hGFAPGFP transgenic mice. Neurosci Lett 513:155-159.

Kim WR, Kim Y, Eun B, Park OH, Kim H, Kim K, Park CH, Vinsant S, Oppenheim RW, Sun W (2007) Impaired migration in the rostral migratory stream but spared olfactory function after the elimination of programmed cell death in Bax knock-out mice. J Neurosci 27:14392-14403.

Kim WR, Park OH, Choi S, Choi SY, Park SK, Lee KJ, Rhyu IJ, Kim H, Lee YK, Kim HT, Oppenheim
RW, Sun W (2009) The maintenance of specific aspects of neuronal function and behavior is dependent on programmed cell death of adult-generated neurons in the dentate gyrus. Eur J Neurosci 29:1408-1421.

Kim WR, Sun W (2013) Enhanced odor discrimination learning in aged Bax-KO mice. NeurosciLett 548C: 196-200.

Kirschenbaum B, Doetsch F, Lois C, Alvarez-Buylla A (1999) Adult subventricular zone neuronal precursors continue to proliferate and migrate in the absence of the olfactory bulb. J Neurosci 19:2171-2180.

Koketsu D, Furuichi Y, Maeda M, Matsuoka N, Miyamoto Y, Hisatsune T (2006) Increased number of new neurons in the olfactory bulb and hippocampus of adult non-human primates after focal ischemia. Exp Neurol 199:92-102.

Marcano-Reik AJ, Blumberg MS (2008) The corpus callosum modulates spindle-burst activity within homotopic regions of somatosensory cortex in newborn rats. Eur $\mathrm{J}$ Neurosci 28:1457-1466.

Rickmann M, Amaral DG, Cowan WM (1987) Organization of radial glial cells during the development of the rat dentate gyrus. J Comp Neurol 264:449-479.

Sanai N, Tramontin AD, Quinones-Hinojosa A, Barbaro NM, Gupta N, Kunwar S, Lawton MT, McDermott MW, Parsa AT, Manuel-Garcia Verdugo J, Berger MS, Alvarez-Buylla A (2004) Unique astrocyte ribbon in adult human brain contains neural stem cells but lacks chain migration. Nature 427:740-744.

Schmidt W, Reymann KG (2002) Proliferating cells differentiate into neurons in the hippocampal CA1 region of gerbils after global cerebral ischemia. Neurosci Lett 334:153-156.

Song JHK, Kang HK, Kim K, Lee SH, Choi D, Cheon MS, Lee Y (2011) Mechanism of ethanol-induced Purkinje cell death in developing rat cerebellum: Its implication in apoptosis and oxidative damage. Dev Reprod 15:205-213.

Sun W, Winseck A, Vinsant S, Park OH, Kim H, Oppenheim RW (2004) Programmed cell death of 
WR Kim, W Sun

adult-generated hippocampal neurons is mediated by the proapoptotic gene Bax. J Neurosci 24:11205-11213.

Yang M, Clarke AM, Crawley JN (2009) Postnatal lesion evidence against a primary role for the corpus callosum in mouse sociability. Eur J Neurosci 29:1663-
1677.

Yu TS, Zhang G, Liebl DJ, Kernie SG (2008) Traumatic brain injury-induced hippocampal neurogenesis requires activation of early nestin-expressing progenitors. J Neurosci 28:12901-12912. 